\title{
Factors Affecting the Perception-Based Compression of Haptic Data
}

\author{
Mehrdad Hosseini Zadeh \\ Kettering University \\ The United States of America \\ David Wang and Eric Kubica \\ The University of Waterloo \\ Canada
}

\section{Introduction}

The ability of technology to transmit multi-media content is very dependent on compression techniques since bandwidth affects how much information can be transmitted in a given amount of time. Researchers have investigated efficient lossy compression techniques for image compression (jpeg) (Miano J., 1999), audio compression (mp3) (Brandenburg K., 1999; Gersho A., 1994) and video compression (mpg) (Bhaskaran V., Konstantinides K., 1999) to facilitate the storage and transmission of audio and video.

Recently, haptics is becoming more important with its addition in various applications such as computer-aided design (CAD), tele-surgery, rehabilitation, robot-assisted surgery, and graphical user interfaces (GUI) to name a few. Haptic technology enables computer users to touch and/or manipulate virtual or remote objects in simulated environments or tele-operation systems. If haptic cues (e.g. touch sensations) are displayed in addition to visual and auditory cues, these VEs are called haptic-enabled virtual environments (HEVEs) (Srinivasan M. and Basgodan C., 1997).

If haptic data is to be stored, transmitted and reproduced, the efficient use of the available bandwidth and computational resources is a concern. Most lossy audio and visual compression techniques rely on the lack of sensitivity in humans to pick up detailed information in certain scenarios. Similarly, haptic perception-based lossy compression techniques utilize limitations in the sensitivity of human touch to create haptic models with much less detail and thus requiring less bandwidth for a given sensation. Essentially, perception-based approaches use the threshold or just noticeable difference (JND) of force perception to develop efficient compression techniques. Force JND is the minimum difference that we can notice between two forces: the base force and an increment/decrement of the base force (Gescheider G.A., 1997). The haptic data would be stored or sent over the network when the value of sampled force data was greater than the force threshold value. It is thus necessary to quantify the force threshold and to investigate the impact of important factors on the force threshold.

Most of the research in this field studied force perception with a human user in static interaction with a stationary rigid object (Hinterseer et al., 2005, 2006). It is equally important to 
measure force JNDs when the user's hand and virtual objects are in motion (Zadeh et al., 2008). This chapter focuses on cases where the human user or the object are in relative motion. In addition, the effects of several factors, including user hand velocity, the base force intensity and the force increment or decrement on force perception are investigated.

This chapter is organized as follows. In Section 2, haptic compression techniques are addressed and perception-based compression techniques are reviewed. Section 3 reviews the sensory threshold of human force perception, Weber's law, several classical psychophysical methods, and previous work on the human haptic system. Section 4 presents an approach to incorporating velocity in the process of measuring the difference force threshold. First, the friction of haptic device is estimated to find the base force of force threshold. Then, an HEVE is constructed to study the effect of user's hand velocity on force perception. The experimental setup and procedure of experiments are described, and the results are presented and discussed. Section 5 studies the effects of the base force intensity and the force increment or decrement on the force threshold. The experimental setup and procedure of experiments are explained in detail, and the results are presented and discussed. Finally, Section 6 summarizes the findings and gives concluding remarks and directions on future research.

\section{Haptic Compression Techniques}

The haptic data compression techniques are divided into two main categories: statistical (Shahabi et al., 2002) and perception-based approaches (Hinterseer P. and Steinbach E., 2005). Statistical approaches mostly focus on the properties of the haptic signal. In contrast to the statistical approaches, perception-based approaches decrease the number of packets using a distortion metric based on the limitations of the human haptic system.

Ortega and Liu in Chapter 6 of Touch in Virtual Environments (McLaughlin et al., 2002) proposed a statistical method that employed similar approaches to those used in speech coding to analyze haptic data. They developed compression techniques that are more specific to the haptic data, including a low-delay coding scheme based on differential pulse code modulation (DPCM). They also presented an alternative coding approach that uses the knowledge of the underlying graphical model. Their findings show that they achieve a compression rate of a factor of 10 using the Low-Delay Predictive coding compression technique.

A variety of statistical methods were compared by Shahabi et al. (2002). They presented and evaluated alternative techniques for achieving efficient sampling and compression of haptic data such as the movement, rotation, and force associated with user-directed objects in a VE. They experimentally determined the benefits and limitations of various techniques in terms of the data storage, bandwidth and accuracy. Again, their study does not include perceptionbased approaches. However, they summarized the result of the statistical approaches that might be useful to compare with the perception-based ones.

Hinterseer et al. (2005) proposed a perception-based compression method to decrease the number of packets transmitted in a telepresence and teleaction system. They sent only haptic data over the network when the value of sampled sensor data is greater than a threshold value. The threshold value was determined in a psychophysical experiment. The results show a considerable reduction - of up to $90 \%$ in the packet rate and data rate - without any perceiveable effect on the fidelity and immersiveness of the telepresence system. Later, they extended their psychophysically motivated transmission method for multidimensional haptic data (Hinterseer P. and Steinbach E., 2006). They used an example of a three dimensional haptic interaction that haptic data are only generated and transmitted if the change in haptic variables exceeds 
the JND of the human operator. Similar to their previous work, the approach reduces packet rates by up to $90 \%$ without impairing immersiveness.

Hinterseer et al. (2006) also presented a model-based prediction of haptic data signals that can be used as a haptic compression technique. This technique can be used to compress haptic data in Internet-based multimedia applications such as haptic-supported games and the haptic rendering of VEs. This method works on the basis of the psychophysical properties of human perception. A two-user tele-operation system was set up, including an operator side and a tele-operator side. A signal prediction model was used on both sides that enabled the users to send packets over the network if the current actual signal differs from the predicted signal by a force threshold. The method reduced the packet rate by up to $95 \%$ without impairing immersiveness. Later, Hinterseer et al. (2006) used fast Kalman filters on the input signals combined with model-based prediction of haptic signals.

Stability is one of the main issues in haptic systems. Instability might cause an undesirable feeling to the user and unrealistic interaction with the virtual environment. One of the most important approaches for designing a stable haptic display is the passivity-based (energybased) approach. The extracted energy from the virtual environment can cause unrealistic feelings with severe destabilizing effects. Colgate J.E. and Brown J.M. (1994) have used a passivity-based model to design stable haptic displays. Kuschel et al. (2006) addressed the issue of stability in data compression algorithms that discard unnoticed data. They focused on guaranteed stability or passivity of a system that uses a lossy data reduction (LDR) algorithm. They proposed a classification scheme for a class of LDR algorithms and derived sufficient stability conditions.

Knowledge about the threshold of human force perception is essential in all reviewed perception-based compression techniques. It is thus necessary to investigate the impact of important factors on the force threshold, including the base force intensity, force increment/decrement, and velocity of the user's hand. However, the effects of these factors have not been addressed in the literature. This chapter studies a set of these factors when the user's hand is in motion.

\section{Sensation, Perception and Psychophysics}

In everyday life, we use our senses to interact with the environment. We can see, touch, smell, hear and taste the external world surrounding us through interactions that usually occur with an initial contact between an organism and its environment. Sensation mostly deals with the initial processes of detecting and encoding environmental energy during the interactions. Essentially, our sense organs convert the energy signals from the environment to bioelectric neural codes and send the codes to the brain (Schiffman, H.R., 2000). The cell receptors of the eye receive the light as environmental energy, transform it into bioelectric codes and then transmit the codes to the brain. Sensation not only deals with the study of the biological events such as the reaction of the eye cells to light energy, but also concerns the relation of sensory experiences to the functioning of sense organs.

In addition to sensations, psychological processes are also required to give meaning to the bioelectric neural codes. When we watch television, our eye initially detects a series of images. However, psychological processes enable us to perceive concepts from the images based on our past experiences, memory, or judgment. In other words, psychological processes present the visual events in a meaningful way. Perception deals with these psychological processes that are required to organize, interpret and give meaning to the output of sense organs. Thus, 
the main objective of sensation and perception is to obtain accurate and reliable information about the environment (Schiffman, H.R., 2000).

Psychophysics refers to the methodology of studying perception. The methodologies from psychophysics are used to study perception (Gescheider G.A., 1997). Psychophysical methods enable us to establish a relation between certain features of environmental stimulation and sensory experiences. Discrimination is the most important perceptual problem that has been addressed in psychophysics. This problem involves the measurement of sensory thresholds, or the perceptual limits of the human sense organs (Brisben et al., 1999). In this study, the sensory thresholds of human force perception are measured in an HEVE.

\subsection{Sensory Thresholds}

The discrimination problem involves deciding whether two stimuli are identical or not. In order to find if there is any difference between the two stimuli, the smallest difference between two stimuli should be measured. The difference threshold or just noticeable difference (JND) is a measure of the minimum difference between two stimuli that is necessary in order for the difference to be reliably perceived. The first stimulus is called base stimulus, and the second stimulus is an increment/decrement of the base stimuli. The JND in the direction of stimuli increment is called the upper limen, and the JND in the direction of stimuli decrement is called the lower limen (Gescheider G.A., 1997). In discrimination experiments, the focus is mostly on the difference in the intensity of two stimuli. However, other dimensions of variation, such as frequency, intensity level, or adaptation time, have also been investigated (Gescheider G.A., 1997). Intensity is subjective quantity which can be triggered by different attributes of a stimulus. This study focus on the amplitude of force as force intensity.

In 1834, Weber studied the relationship between the difference thresholds or JNDs and the intensity levels of the base stimulus. He discovered that the JND increases significantly for very small intensities and decreases while the intensity of the base stimulus increases. For relatively large base stimuli, Weber found that the JND is a linear function of stimulus intensity. In other words, the difference threshold is always a constant fraction of the stimulus intensity for those base stimuli; this fraction is called Weber's fraction. This trend is observed by other researchers and is called the Weber trend (Gescheider G.A., 1997). The value of Weber's fraction is different for various senses.

The linear relationship is a valid law for all senses and sense organs. This relationship is called Weber's law, which can be represented as

$$
\Delta \phi=c \phi \text { or } \Delta \phi / \phi=c,
$$

where $\mathrm{c}$ is the constant Weber's fraction, $\Delta \phi$ is the change in the stimulus intensity that can just be noticeably different (JND), and $\phi$ is the starting intensity of the stimulus or base stimulus.

\subsubsection{The Force Thresholds of the Human Haptic System}

Srinivasan M. and Basgodan C. (1997) defined the human haptic system as the entire mechanical, sensory, motor and cognitive components of the body-brain system. Researchers have determined the force thresholds of the human haptic system in real world situations ((Jones $\mathrm{L}$. A., 1989);Pang et al., 1991; Raj et al., 1985). Jones L. A. (1989), in a force matching experiment focused on a human elbow, found a JND ranging between $5 \%$ and $9 \%$ over a range of different base force values. Subjects were required to generate forces ranging from 15 to $85 \%$ of their maximum voluntary contraction (169-482 N). Pang et al. (1991) determined a JND that lies between $5 \%$ and $10 \%$ for pinching motions between finger and thumb with a constant resisting 
force. This JND was found to be relatively constant over a range of different base force values between 2.5 and $10 \mathrm{~N}$. Raj et al. (1985) studied the ability of human subjects to discriminate between different magnitudes of weights. They found JNDs of $12 \%-13 \%$ for large base weights (80-200 g) lifted by the middle finger about the metacarpophalangeal (MCP) joint.

Allin et al. (2002) measured force JND in a VE. The goal was to use the force threshold to construct therapeutic force feedback distortions that stay below the threshold. The focus was on JND as applied to the index finger. The result was an average JND of approximately $10 \%$ over a number of subjects with a constant base force at $2.25 \mathrm{~N}$. The conclusion was that the visual feedback distortions in a VE can be created to encourage the increment of force production by up to $10 \%$, without a patient's awareness.

\subsubsection{Force Thresholds and Motion}

In the previous subsection, the reviewed studies have measured the force thresholds in the haptic display of stationary rigid objects, which interact with the operator's hand. However, motion is critical in many VR applications.

Very little research has considered the study of motion and perception in the haptic displays (Lederman et al. (1999); Jandura L. and Srinivasan M. A. (1994)). Lederman et al. (1999) investigated the effects of the speed of the relative motion on perceived roughness via a rigid probe. Several experiments were conducted based on the mode of touch, active or passive, and different ranges of velocities. It was realized that the effects are multiple and complex. The results show that increasing speed tended to render surfaces as smoother. It was also observed that the inter-element spacing for texture perception has a significant effect in addition to changes in the speed. In other words, perceived roughness decreases with increasing speed, up to the point where the probe tip is able to fall between the inter-element spaces, where the effect is reversed. This chapter also focuses on the effects of the relative velocity on the human haptic perception. However, the goal is to explore the limitations of the haptic perception in the haptic rendering of VEs.

Jandura L. and Srinivasan M. A. (1994) conducted torque discrimination experiments for a slow twisting motion. Subjects were asked to maintain a constant angular velocity, while a constant torque was applied on the subjects' hands. The results show that the JND for torque was $12.7 \%$ when the reference torque was $60 \mathrm{mN}-\mathrm{m}$.

\subsubsection{Psychophysical Methods for Measuring Thresholds}

There are many methods to determine the absolute and difference thresholds. According to Gescheider (Gescheider G.A., 1997), methods of limits, constant stimuli, and adjustment are among the most well known methods for detecting absolute and difference thresholds. People are usually presented with the same stimuli on different occasions. However, they do not always respond in the same ways. The main reason for this is presumably that the neurosensory system allows a margin of error. Other sources of biases such as learning and adaptation, can also be a factor.

One of the best techniques for detecting sensory thresholds is the method of limits and it is not as time consuming as other methods. In this method, a subject is presented with a stimulus well above or below the expected threshold. On each trial, the subject indicates detection of the stimulus with a yes response, or non-detection with a no. The experimenter increments the stimulus on successive trials if the first stimulus presented is below the threshold, until the subject changes his response from no to yes. If the first stimulus is over threshold, the stimuli are gradually decremented in steps until the subject's response changes from yes to no. 


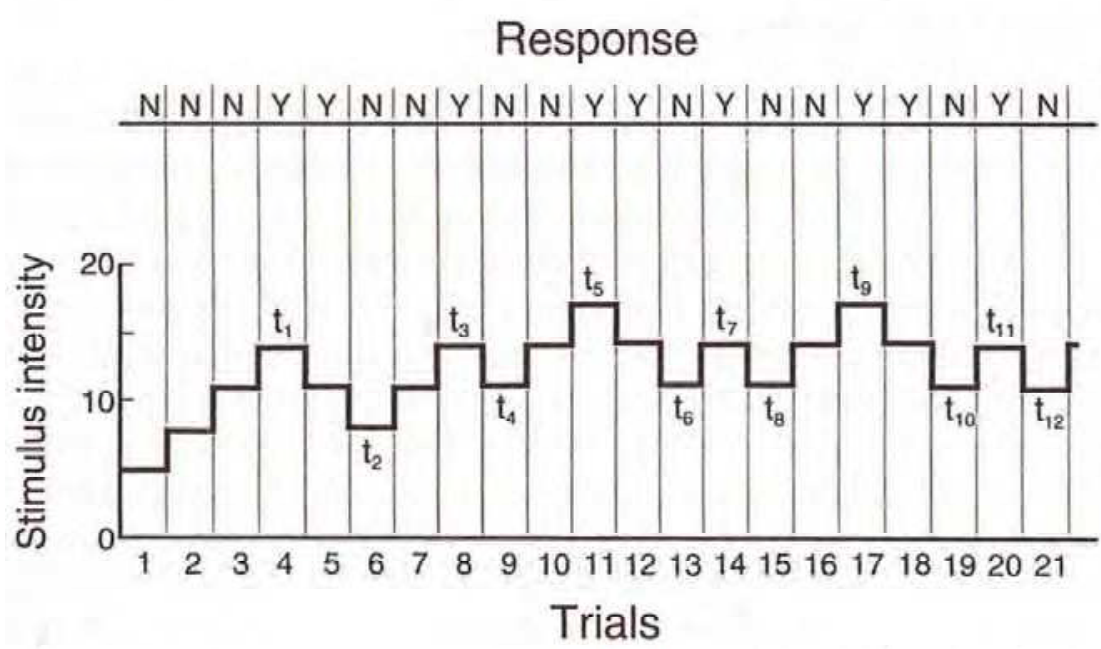

Fig. 1. Staircase method: yes $(\mathrm{Y})$ or no $(\mathrm{N})$ responses are shown in the top of figure. The transition points are indicated above and below of stairs Gescheider G.A. (1997).

A series is terminated immediately after the first change in response, and the transition point for that series is taken as the stimulus value halfway between the last two stimulus values. Several ascending and descending series can be conducted, and the absolute threshold is the average of the transition points over all of the series.

Another effective method is the staircase method which is a modification of method of limits for detecting absolute thresholds. It is very similar to the method of limits with the only difference being that each series does not terminate after a transition point, and the direction of the series is reversed. As shown in Figure 1, if the stimulus is being incremented, after the first yes response it will begin to be decremented, and vice versa.

The procedure is finished when a sufficient number of response transition points have been recorded. The result of averaging the transition points is the threshold. This method takes less time compared to other methods because only a few stimulus values that are far above or below threshold are presented. Although it is a very efficient method, its sources of biases are the same as the method of limits Gescheider G.A. (1997).

The Interweaving Staircase (IS) (Bernstein R. S., and Gravel J. S., 1990) method is a variation of the staircase method that is used to measure the JND in the direction of force increment and in the direction of force decrement. In the IS method, the experimenter starts by presenting a sequence of forces which is the base force plus the increment or decrement, then progressively increases or decreases in value. The subject responds with yes to increment or decrement or no to detecting changes in force value. When the subject's response changes from one or a series of the same response to the other response, as is the case of yes, yes, yes switching to no, the force value is recorded, and the direction of the force sequence is reversed from ascending to descending, or vice versa. These points are called transition points. The transition points are recorded and the JND value is the average value of the transition points. 
The main advantage of the IS method is to reduce the possible biases compared to the original staircase method. In the IS method, a subject has to report one of the three possible responses, increment, decrement, or no change. Therefore, it is much more difficult for the subject to guess the response, and it is possible for the experimenter to check the response with the current direction of force whether ascending or descending.

\section{The Effect of Velocity on Force Perception in HEVEs}

This section reports the results of a pilot study that is conducted to investigate the relation between motion and human haptic perception. We study the effects of a user's hand velocity on force perception in an HEVE. The focus is on the determination of difference force threshold or JND by measuring the upper and lower limens of force JND. The force JND is obtained in the free motion condition of the PHANToM device when the device end-effector is grasped by a subject's hand. In free motion, there is no interaction with virtual objects, and no force feedback is applied on the subject's hand. The only force on the user's hand is a resistive force due to the backdrive friction of the device. Thus, this friction force is the base force for the force JND when no force feedback is applied. In the next section, Section 5, a full study is conducted to measure force JNDs for three base forces when force feedback is also applied on the subject's hand.

In two experiments, subjects are asked to report the just noticeable difference between the base force and an increment/decrement from it when they perceive the JND. The upper and lower limens of the force JND are quantified for three ranges of velocity: low $(0.03-0.05 \mathrm{~m} / \mathrm{s})$, medium $(0.12-0.15 \mathrm{~m} / \mathrm{s})$, and high $(0.22-0.28 \mathrm{~m} / \mathrm{s})$.

The experiments are described in detail in Section 4.2. The upper and lower limens of force JND for the three ranges of velocity are presented in Section 4.3 and discussed in Section 4.4.

\subsection{Hypothesis}

4.1.1 $\mathrm{H}_{1}$ :

The force JND of human force perception increases when the velocity of user's hand increases in an HEVE.

\subsection{Methods}

This section describes the two conducted experiments, which use the same setup, task, and procedure. The direction of applied forces is the only difference between the two experiments. In the first experiment, the applied force is in the same direction of hand motion (aid force). Thus, the force partially cancels the friction, and decreases the resistive force. In the second experiment, the applied force is in opposition to the direction of the subject's hand motion (opposed force) and increases the resistive force.

\subsubsection{Participants}

There were eight right-handed participants who were between the ages of 27 and 34 . All were regular computer users and students at the University of Waterloo. Participation was voluntary. The participants did not have any neurological illness or physical injury and had no more than trivial previous exposure to haptic interfaces. The experiment was conducted in accordance with the University of Waterloo ethical guidelines. 


\subsubsection{Apparatus}

The PHANToM ${ }^{T M}$ Omni device was used in both experiments. This haptic device has been designed for a vast variety of applications, including medical, scientific and industrial. In general, some of the advantages of PHANToM device are their 3D force-feedback, the ability to operate in an office or desktop environment, compatibility with standard PCs and useful for a broad range of applications.

Omni devices have a relatively large workspace for desktop applications, suitable for a large range of hand motions, stiffnesses and motor forces to meet the specific requirements of this research project. Comparing to other haptic devices, this device is widely used in various applications because of its reasonable price. This device can generate maximum $3.3 \mathrm{~N}$ force, which is enough for the purpose of the experiments. The applied forces in the experiments in this study are less than $1 \mathrm{~N}$. Another important characteristic of the device is the backdrive friction, which is reported as up to $0.26 \mathrm{~N}$ (Sensable Technologies Inc.). In Section 4.2.2.1, this friction and its variability are estimated for the part of work space that is used in the experiments. Essentially, the variability is important because we need to know the minimum detectable force output of the device.

The haptic device is connected to a personal computer through a Firewire interface card. The software has two processes, haptic and graphic, that are run on two $3 \mathrm{GHz}$ Pentium $4 \mathrm{com}-$ puters running Windows XP. Force feedback is generated by the haptic process. The graphic process renders a 2D VE that is shown in Figure 2. The VE contains a colour ball and a colour bar. The colour ball represents the position of the device end effector (grasped by the subject's hand). The bar is stationary and has two green ends (targets). The center-to-center distance between the targets on the bar is $10.2 \mathrm{~cm}$. As shown in Figure 3, a 17" LCD monitor, which is placed approximately $70 \mathrm{~cm}$ from the subject, is used to display the VE. The update rate of haptic (force) display is $1000 \mathrm{~Hz}$.

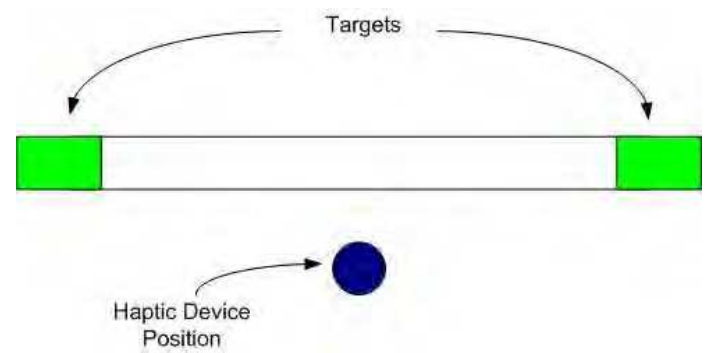

Fig. 2. The 2D VE that contains a 2D ball and a bar with two green target zones.

\subsubsection{Estimation of the Device Friction}

In the experiment, the base force is the resistive force due to the friction of the haptic device, which includes coulomb and viscous (damping) friction. Coulomb or dry friction is independent of velocity, however, viscous friction is proportional to the velocity of device end-effector (Berkelman, P. and Ji M., 2006), and is usually reported as a coefficient in Ns/m. Similar to coulomb friction, viscous friction is calculated in $\mathrm{N}$ when viscous damping coefficient is multiplied by velocity $(\mathrm{m} / \mathrm{s})$. 


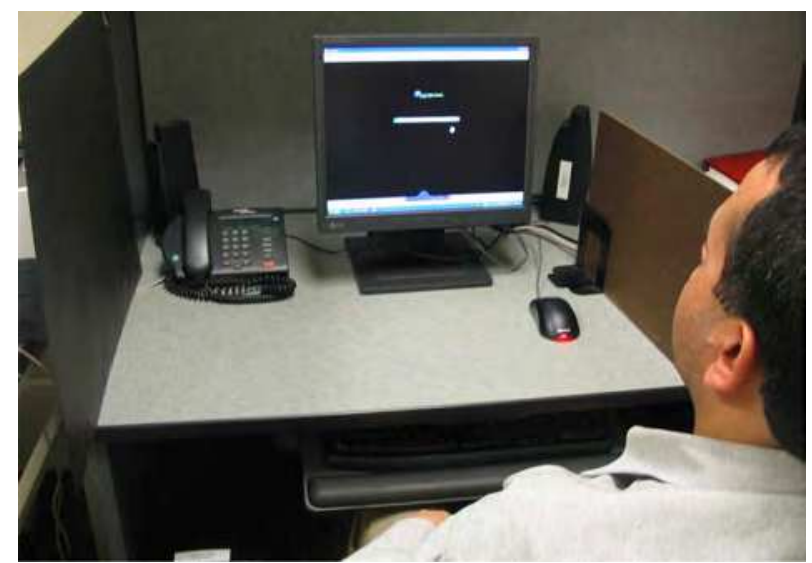

Fig. 3. The computer display shows the 2D VE.

The viscous frictions of PHANToM devices are very small because a cable-pulley transmission is used in these devices. Diolait et al. (2005) found that the coulomb friction is $0.038 \mathrm{~N}$ and the viscous friction coefficient is $0.005 \mathrm{~N} . \mathrm{s} / \mathrm{m}$ for PHANToM 1.0 haptic device. Thus, if the end-effector moves by $0.28 \mathrm{~m} / \mathrm{s}$, the viscous friction is $0.0014 \mathrm{~N}$, which is negligible compared to the coulomb friction. Their measured friction is similar to the backdrive friction of the PHANToM 1.0, up to $0.04 \mathrm{~N}$, as reported in the device specifications (Sensable Technologies Inc.).

The backdrive friction of the PHANToM Omni is reported up to $0.26 \mathrm{~N}$, which is 6.5 times larger than the friction of PHANToM 1.0. Therefore, the Omni viscous friction is approximately $0.009 \mathrm{~N}$ if the end-effector of Omni moves by $0.28 \mathrm{~m} / \mathrm{s}$, which is the maximum velocity in the experiments. Thus, in all experiments, it is assumed that the resistive force is only due to the coulomb friction of the device, which is basically independent of velocity.

In addition, the preliminary experiments showed that the friction was variable within the workspace of the device. In other words, when we moved the device end-effector manually, we had to change the force intensity to maintain the motion with a constant velocity. Therefore, the friction and its variability were estimated within the part of workspace that was used in the experiments. The workspace was a $10.2 \mathrm{~cm}$ path, from -0.051 to $0.051 \mathrm{~m}$ on the $\mathrm{x}$-axis of the device workspace.

In order to determine the friction, varying force profiles were applied to: a) move the endeffector from a static condition and b) keep an approximately constant velocity of the endeffector from the beginning to the end of the path. The applied force in $b$ ) should be equal to the friction if the force produces an equilibrium trajectory (the end-effector moves with a constant velocity on the whole path).

Initially, the end-effector was placed at a point $(0.07 \mathrm{~m}$ on $\mathrm{x}$-axis) before the beginning of the path. A relatively high intensity force was applied to the end-effector for $400 \mathrm{~ms}$ to move the end-effector toward the beginning of the path ( $0.051 \mathrm{~m}$ on $\mathrm{x}$-axis). Finally, a weaker force was applied to the end-effector and this force was maintained until the device reached the end of the path.

Since the friction was variable, two frictions were estimated when the end-effector moved from right to left and left to right. To estimate the right to left friction, first, forces with different 
magnitudes were applied to find the force that could overcome the static friction. A $0.5 \mathrm{~N}$ force could initially move the end-effector, and overcome the static friction. Then, a weaker force of $0.26 \mathrm{~N}$ was applied to maintain a constant velocity (around $0.16 \mathrm{~m} / \mathrm{s}$ ). Figure 4 shows the trajectory of the end-effector when the force profile was applied. For the first $1000 \mathrm{~ms}$, no force was applied, and then $0.5 \mathrm{~N}$ was applied for $400 \mathrm{~ms}$ followed by $0.26 \mathrm{~N}$ for $1600 \mathrm{~ms}$.

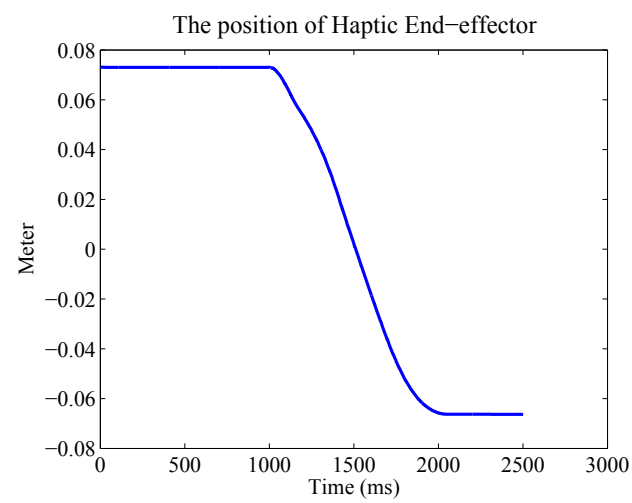

Fig. 4. The end-effector trajectory when no force was applied for $1000 \mathrm{~ms}$, then a $0.5 \mathrm{~N}$ force was applied for $400 \mathrm{~ms}$ followed by a $0.26 \mathrm{~N}$ for $1600 \mathrm{~ms}$.

To estimate the left to right friction, the force to overcome the static friction was $0.31 \mathrm{~N}$, which was less than the applied force for the right to left. However, the force applied to maintain a constant velocity was slightly higher at $0.28 \mathrm{~N}$.

Based on the results, the friction was $0.27 \pm 0.01 \mathrm{~N}$, which was the average of the two weaker forces for the right to left and left to right movements.

Other researchers ignored the friction force in their base force because the friction force is negligible compared to large base forces applied by the actuators such as $2.25 \mathrm{~N}$ in Allin's work, 2002. However, in this study, the small friction force should be taken into account because our base forces are small.

\subsubsection{Design}

In this pilot study, a mixed model design was used in the experiments (Reis \& Judd, 2000). This model involves both a within-subject design as well as a between-subject design. In the mixed model design, several independent variables can vary within subjects and other variables can vary between subjects. The conducted experiments were devised to measure the force JNDs in a velocity-based scenario. The dependent variable was the force JND of human force perception. The independent variables were the velocity of subject's hand motion and the force increment/decrement. All participants experienced all three levels of the velocity because the main goal is to investigate the effect of the velocity. However, four subjects experienced the force increment or the force decrement since four subjects were enough to determine one limen of force JND. Participants were divided into two groups of four. One female and three male subjects were in each group. The first group participated in the experiment for the force increment and the second group participated for the force decrement. The order of the experiments and levels randomly assigned to the subjects. 


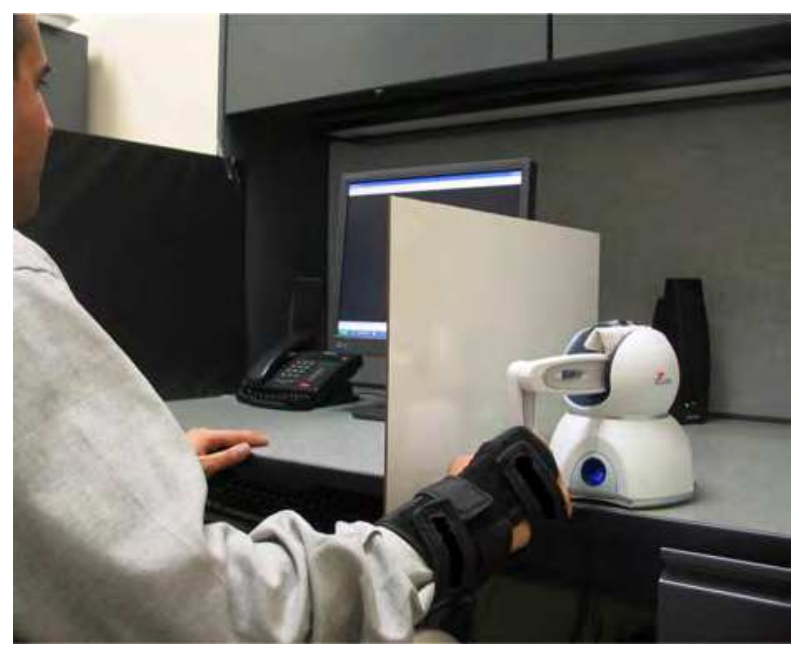

Fig. 5. The subject grasps the end-effector of the PHANToM ${ }^{T M}$ Omni and rotate his hand about his elbow.

\subsubsection{Procedure}

Each subject is seated on a chair facing a computer display and asked to place their right elbow on a side support. The wrist of the right hand is restrained with a wrist guard, as shown in Figure 5, so that wrist movements are locked to ensure that subjects just rotate their hands about their elbows. The subject grasps the end-effector of the haptic device.

During each experiment, the attention of the subject is directed to the display containing the $2 \mathrm{D} V E$, as shown in Figure 2. The ball represents the device end-effector and moves when the subject moves the end-effector. The right arm and fingers of subjects are shielded from their own view with an opaque barrier to ensure subjects control their hands' movement via visual feedback from the display. The subject is asked to move his/her hand, back and forth, from left to right and then right to left, repeatedly. The subject is required to maintain the red ball between the green zones and not go beyond the zones.

Three ranges of reference velocity are selected based on two factors. First, the ability of subjects to carry out the experimental task at the velocity ranges. Second, having relatively large rooms among the ranges to study any potential significant difference of the force JNDs at the ranges. The selected ranges are low $(0.03-0.05 \mathrm{~m} / \mathrm{s})$, medium $(0.12-0.15 \mathrm{~m} / \mathrm{s})$, and high $(0.22$ $-0.28 \mathrm{~m} / \mathrm{s}$ ). To find the ranges at which subjects could complete the task, several subjects, other than the main eight subjects, carried out the task within various velocity ranges before starting the main experiments.

All subjects are required to maintain their hand velocity within the specified ranges in three different experiments. The colour of the ball in the display aids the subjects in maintaining the average value of their hands' velocity at the reference velocity. If the subject's velocity is within the range of reference velocity, the ball's colour is red. Otherwise its colour is yellow. Therefore, the subjects control their hands' velocity by observing the ball's colour. To ensure that subjects can control the velocity, they are given training before starting the main experiments. 


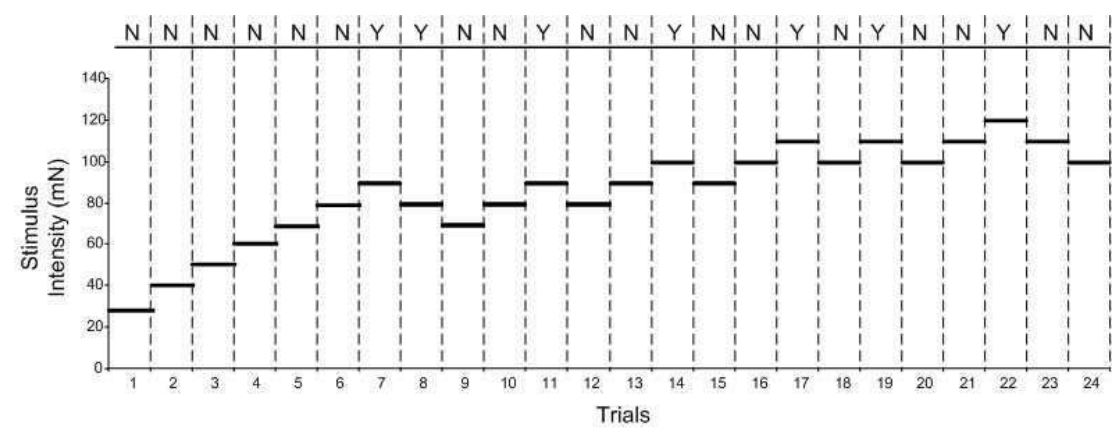

Fig. 6. The staircases of the applied forces. (no: N; yes: Y)

The average velocity is used because the subject stops at the end of the bar and moves towards the other side of the bar in the 2D VE, as shown Figure 2. If the velocity at each instant in time is used, then the ball's colour would turn to yellow at the end of the bar, and the subject might inadvertently apply extra force. This can distract the subject and affect the process of measuring the force JNDs. Thus, the mean velocity value is used as it does not change rapidly when the subject stops at the end of the bar, and the ball's colour does not turn to yellow.

The staircase method, which is explained in Section 3.1.3, is used to measure the force JNDs. In the middle of each trial, the experimenter applies a certain amount of either opposed or aid force to the subject's hand motion based on the staircase procedure. Each subject is asked to report any changes in the haptic sensations on their hand during each trial. Before applying the force, the experimenter ensures that each subject maintained the hand velocity within the reference range. The procedure is finished when 12 transition points are obtained. Therefore, the number of trials is variable. As a results, the duration of experiment is variable, and one experiment typically takes from 15 to 25 minutes. Twelve transition points are recorded and the force JND value is the average value of the transition points.

This procedure is repeated in three sessions for the three velocity ranges, low, medium, and high. Figure 6 shows the staircases of the force JND for one of the subjects in an experiment. Each subject is given training. The first session includes a familiarity phase and then the experiment is conducted for one of the velocity levels. In the second session, the subject does the same task with another velocity level. In the last session, the same task is done with the last velocity level.

The zero velocity is detected in the application of forces. The issue is encountered when the direction of motion frequently changes during a trial. For example, if the subject moves from left to right, the end-effector's velocity is positive. When the subject stops at the target and moves from right to left, the velocity's sign changes to negative, leading to a zero velocity at the targets. As a result, several sudden changes occur in the applied force, causing the device to switch discontinuously and jittering movement.

To overcome this problem, a model is developed using a narrow dead-zone as shown in Figure 7. In this model, if the velocity is within the velocity interval $(\mathrm{dV})$, the applied force is set to zero by the dead-zone (dV). For these experiments, the $|\mathrm{dV}|$ of $0.001 \mathrm{~m} / \mathrm{s}$ is found to be sufficient to solve the problem. Our model is similar to the Karnopp (1985) model, which represents friction force at zero velocity. 


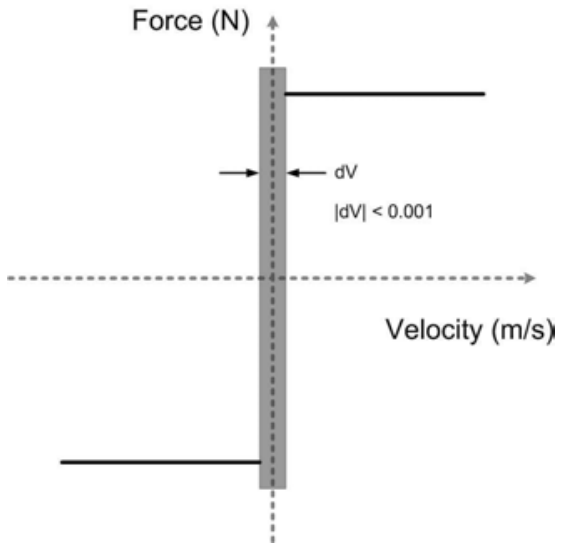

Fig. 7. The zero velocity detection model: no forces are applied on the subjects' hands when the velocity is within the interval $\mathrm{dV}$.

\subsection{Results}

The average force JNDs across all subjects and for all levels of the velocity and force increment/decrement are shown in Table 1 and Figure 8. For example, the upper and lower limens of force JNDs are $18.91 \%(0.051 \mathrm{~N})$ and $18.36 \%(0.049 \mathrm{~N})$ when the subjects' hands are in the low velocity motion.

\begin{tabular}{|c|c|c|c|}
\hline $\begin{array}{c}\text { Rang of velocity } \\
(\mathrm{m} / \mathrm{s})\end{array}$ & $\begin{array}{c}\text { Force } \\
\text { Increment }\end{array}$ & $\begin{array}{c}\text { Force } \\
\text { Decrement }\end{array}$ & Average \\
\hline Low $(0.03-0.05)$ & $18.91 \pm 1.43$ & $18.36 \pm 0.9$ & $18.63 \pm 0.79$ \\
\hline Medium $(0.12-0.15)$ & $27.08 \pm 2.93$ & $26.23 \pm 2.85$ & $26.66 \pm 1.90$ \\
\hline High $(0.22-0.28)$ & $36.19 \pm 4.3$ & $32.95 \pm 3.00$ & $34.57 \pm 2.51$ \\
\hline Average & $27.39 \pm 2.68$ & $25.85 \pm 2.21$ & \\
\hline
\end{tabular}

Table 1. The average force JNDs $(\%)$ of base friction force $(0.27 \mathrm{~N})$ and standard errors for all levels of the velocity and force increment/decrement across all subjects. The average JND of four subjects are included in each cell.

As shown in Figure 8, the average force JND values are in a range between $18.91 \%$ to $36.19 \%$ for force increment and $18.36 \%$ to $32.95 \%$ for force decrement, indicating that the force JNDs increase when the subject's hand velocity increases.

For the low velocity, the upper and lower limens are almost equal. However, the difference between the upper and lower limens slightly increases by increasing the velocity. The upper limen is $4 \%$ higher than the lower limen for high velocity.

To study the effect of the velocity and force increment/decrement, a two-way analysis of variance (ANOVA) is conducted. Statistical analysis is performed using a mixed (within and between-subject) analysis of variance (ANOVA) with $\mathrm{p}<0.05$ as the rejection level.

The average of force JNDs are calculated for the three ranges of velocity across the two levels of force increment/decrement and shown in Figure 9. This figure also shows that the velocity 


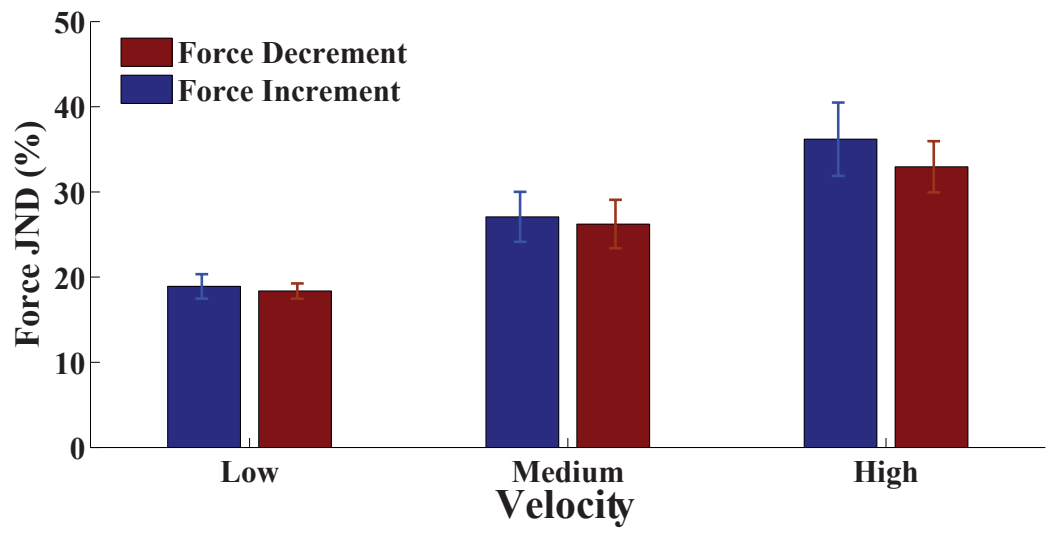

Fig. 8. The average of force JND across all subjects and standard errors for the three levels of velocities and the two levels of force increment/decrement. The results of ANOVA significantly supports the trend of increasing the force JND when the velocity increases, $\mathrm{p}<0.0001$.

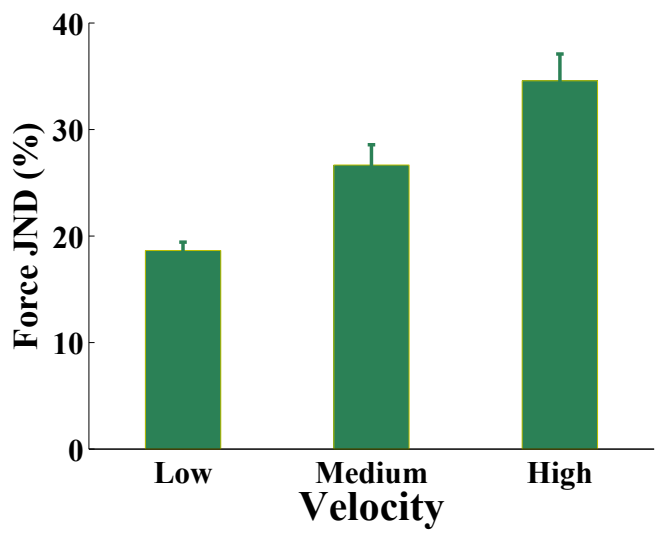

Fig. 9. The mean values and standard errors of force JND for the three ranges of velocity across the force increment and decrement. 


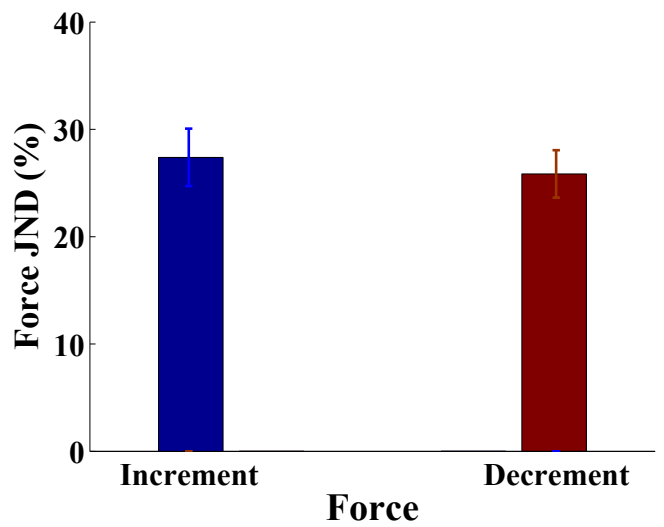

Fig. 10. The mean values and standard errors of force JND values across all ranges of velocity. The results of ANOVA also show no statistically significant difference between the force increment and decrement, $\mathrm{p}=0.6804$.

of the subject's hand has a significant effect on the force perception of the subject. For example, the force JND for high velocity is almost twice as large as the low velocity force JND with very small standard errors. The results of ANOVA significantly supports the trend of increasing the force JND when the velocity increases in Figures 8 and 9, $F(2,12)=56.75$ and $p<0.0001$. The average of force JND were also calculated for the two levels of force increment and decrement across the three ranges of velocity and shown in Figure 10. As shown in the figure, the force increment and decrement are almost equal for all velocities, indicating that the upper and lower limens of force JNDs are somewhat symmetric. The results of ANOVA, $F(1,6)=$ 0.19 and $\mathrm{p}=0.6804$, also show no statistically significant difference between the force increment and decrement.

\subsection{Discussion}

An HEVE is constructed to study the effect of a user's hand velocity on force perception. An approach is presented to incorporate the velocity in the process of measuring the force perception threshold. The force JNDs are measured for three ranges of velocity. The trend of data, which is significantly supported by the results of an ANOVA, confirmed the hypothesis $H_{1}$, indicating that the force JND increases as the velocity of the user's hand increases in an HEVE.

The results also indicate that the upper and lower limens of force JND are almost equal for low and medium velocities, and the upper limen is slightly larger than the lower limen for high velocity motion. The results of an ANOVA also do not show any significant difference between the limens. In Section 5, the difference between limens will be investigated for smaller and larger base forces.

The results show that the measured force JNDs are larger than the JNDs measured by Pang et al., (1991) and Jones L. A. (1989), who determined the force JND in a range of $7 \%-10 \%$ for different muscle groups in hand and arm under various conditions. For example, Pang et al., (1991) found a JND that lies between 5\% and 10\% for pinching motions with a constant resisting force over base forces between 2.5 and $10 \mathrm{~N}$. 
Similarly, the force JNDs are higher than the JND measured in a VE by Allin et al., (2002), who found a $10 \%$ force JND on the index finger with a constant base force at $2.25 \mathrm{~N}$. On the other hand, the low velocity JNDs are compareable to the JNDs obtained in a VE by Brewer et al., (2005) who found a $19.7 \%$ force JND for a $1.5 \mathrm{~N}$ base force. They also reported that their JND is larger than the JND in the literature, discussing several reasons such as the difference in the environment and tested joint, less subjects' training, and unfixed background dimensions. In this study, the small base force $(0.27 \mathrm{~N})$ can be the main reason that the JNDs are larger than the JNDs measured by Pang et al., (1991); Jones L. A. (1989); and Allin et al., (2002). The base force is much smaller than their base forces $(1.5-10 \mathrm{~N})$, and according to Weber's law, the JNDs for low base stimuli are larger than ones for high base stimuli. In the next section, Section 5, this difference will be investigated with different base forces to find if the higher JND in this section is due to a small base force.

\section{The effect of force intensity on Force Perception in HEVEs}

In this section, the force JNDs of the human haptic system are quantified and the effects of the base force intensity and the force increment/decrement on the force JND are investigated. An experiment is conducted for three levels of base force intensity. The Interweaving Staircase (IS) Bernstein R. S., and Gravel J. S. (1990) method is employed to measure the force JNDs. For the first level, $0.15 \mathrm{~N}$ force is applied in the same direction as the hand motion to partially cancel the backdrive friction of the haptic device $(0.27 \mathrm{~N})$. Therefore, the resulting resistive force on the subject's hand is $0.12 \mathrm{~N}$, which is the first level of base force. This level is called the low base force.

For the second level, $0.15 \mathrm{~N}$ is applied in opposition to the direction of the hand motion. This force adds to the friction force, resulting in a $0.42 \mathrm{~N}$ resistive force on the subject's hand. This force is called the medium base force.

For the third level, $0.5 \mathrm{~N}$ is applied in opposition to the direction of subject's hand motion, resulting in $0.77 \mathrm{~N}$ force on the user's hand. This is called the high base force. Thus, the three resultant base forces are $0.12 \mathrm{~N}$ (low), $0.42 \mathrm{~N}$ (medium), and $0.77 \mathrm{~N}$ (high); two greater than the friction and one smaller.

The hypotheses are presented in the next subsection, and the experiment is described in detail in Section 5.2. The results are presented and discussed in Section 5.3.

\subsection{Hypotheses}

In Section $4, H_{1}$, which is that the force JND increases when the velocity of user's hand increases, was tested. In this section, the following hypotheses are proposed and tested. These hypotheses are based on the results in the Section $4 . \mathrm{H}_{2}$ is examined to investigate why the measured force JNDs in Section 4 were larger than the JNDs in the literature. $H_{3}$ is tested to find any significant difference between the upper and lower limens of force JND for a relatively high velocity motion and different base forces.

\subsection{1 $\mathrm{H}_{2}$ :}

Weber's law holds for force perception in an HEVE even when the user's hand is in motion. The force JND is larger for very small base force intensities and decreases as the base force intensity increases. 


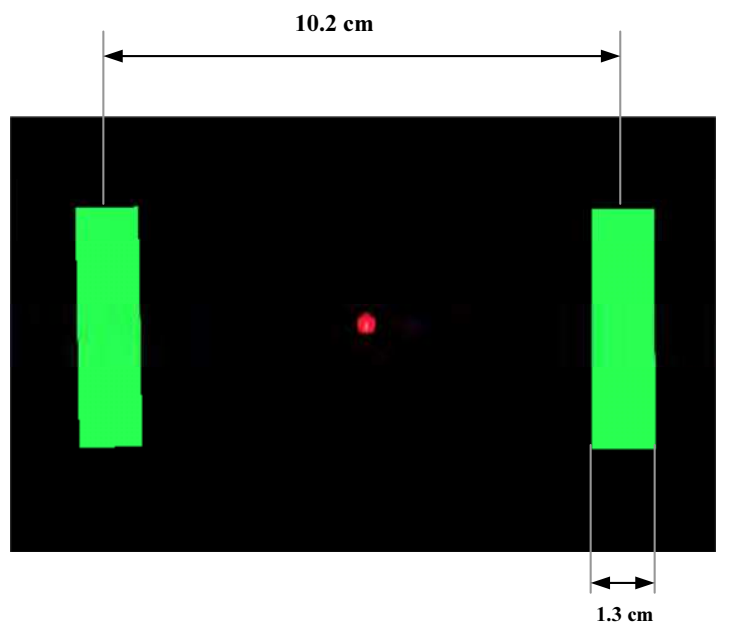

Fig. 11. The 2D virtual environment that contains a red 2D ball and two green rectangles.

\subsection{2 $\mathrm{H}_{3}$ :}

The upper and lower limens of force JND are not symmetric for all base forces when a subject's hand is in motion.

\subsection{Methods}

The experimental setup and procedure of the experiment are described. The intensity of the base force is the only difference between the three levels of the experiment.

\subsubsection{Participants}

There were 16 paid right-handed participants (eight females and eight males) between the ages of 22 and 33. All were regular computer users and students at the University of Waterloo, and had no neurological illness or physical injury that would impair hand function or force control ability. The experiment is conducted in accordance with the University of Waterloo ethical guidelines.

\subsubsection{Apparatus}

In this experiment, the same haptic device and monitor are used as in the previous section. A $2 \mathrm{D}$ VE is created and graphically rendered to users through the 17" LCD monitor. The VE is haptically rendered to subjects via the haptic device. As shown in Figure 11, the VE contains a $2 \mathrm{D}$ red ball and two green rectangles (targets). The center-to-center distance between the targets is $10.2 \mathrm{~cm}$, and the width of the target is $1.3 \mathrm{~cm}$ in display coordinates and in the haptic device space. The ball represents the position of the end effector (grasped by the subject's hand). When the subject moves the end-effector, the ball moves on a horizontal line.

\subsubsection{Design}

A Repeated Measures (within subject) design Kuehl R. O. (2000) is employed in this experiment. Therefore, each subject is required to participate in all levels of the experiment plus a one-hour training session. The order of levels are randomly assigned to the subjects. 
The base force intensity and the force increment/decrement are the independent variables. The base force intensity have three levels; low, medium, and high. The force increment/decrement factor have two levels based on the relative changes from the base force. At the half of trials in each base force level, the force increases from the base force, and at the other half, force decreases. The two levels of force increment/decrement are called increment and decrement.

\subsubsection{Procedure}

Similar to the experiments in Section 4, the subject is seated on a chair facing the monitor and asked to place their right elbow on a side support. The wrist of the right hand is restrained with a wrist guard, so that wrist movements are locked (to ensure that the subject just rotates his/her hand about his/her elbow). The subject grasps the device end-effector. Once the subject is seated comfortably, his/her right arm and fingers are shielded from his/her own view with an opaque barrier. Attention is directed to the monitor, which is placed approximately $70 \mathrm{~cm}$ from the subject.

As shown in Figure 12, each trial begins and ends with verbal commands (start and stop). Subjects start a task when they hear start from the experimenter. The task is explained later in Task subsection. Subjects stop and let go of the end-effector when they hear stop, and wait for 5-15 seconds before starting the next trial. During that time, no force is applied on the subject's hand, and the subject's hand is not in motion. Each trial has two intervals, and each interval lasts 15 seconds.

Figure 12 shows the first three trials of an experiment. In trial 1, the first force (F) is continuously applied on the subject's hand from the beginning of the trial until the end of the first interval. This force is $0.15 \mathrm{~N}$ in the same direction as the hand motion for low base level. The force vector is shown in Figure 13 as the aid force. The force is a $0.15 \mathrm{~N}$ for medium and a 0.5 $\mathrm{N}$ for high base levels. These forces are in opposition to the direction of the hand motion, and shown in Figure 13 as the opposed force. The relative direction of applied force to the hand motion does not change during an experiment.

At the beginning of the second interval, the second force is applied to the subject's hand. This force is either an increment or decrement from the first force. The trials with a force increment and decrement called force increment and force decrement trials, respectively. Each base force level of the experiment consists of 48 trials: 24 increment and 24 decrement trials. The order of trials was randomly chosen by the experimenter before starting the experiment.

The subjects are asked to detect changes in force value at the end of each trial. They respond with yes if they sense a force increment or a force decrement. They respond with no if they do not notice any changes. The trials with no and yes responses are called unnoticed and noticed trials, respectively. For instance, trial 1 is an unnoticed trial because the subject's response is No.

The force is increased/decreased by $0.02 \mathrm{~N}$ in the first trial of both force increment and decrement trials. The values of the force increment/decrement in next trials are determined based on the response of the subject in the current trial. Two variables are initialized by $0.02 \mathrm{~N}$. One variable, which is called $\Delta \mathrm{F}_{I n c}$, saves the increment value for the next force increment trial, and the other one, $\Delta \mathrm{F}_{\text {Dec }}$, saves the force decrement value for the next force decrement trial. These variables would increase by $0.01 \mathrm{~N}$ if the response was No, and would decrease by 0.01 $\mathrm{N}$ if the response was yes. For example, $\Delta \mathrm{F}_{\text {Dec }}$ value for trial 2 is $0.03 \mathrm{~N}(0.02+0.01)$ because the response is no in trial 1. Therefore, $0.15 \mathrm{~N}$ decreases by $0.03 \mathrm{~N}$, and $0.12 \mathrm{~N}$ is applied at the second interval of trial 2. $\Delta \mathrm{F}_{\mathrm{Dec}}$ value would decrease by 0.01 if the response was yes. 


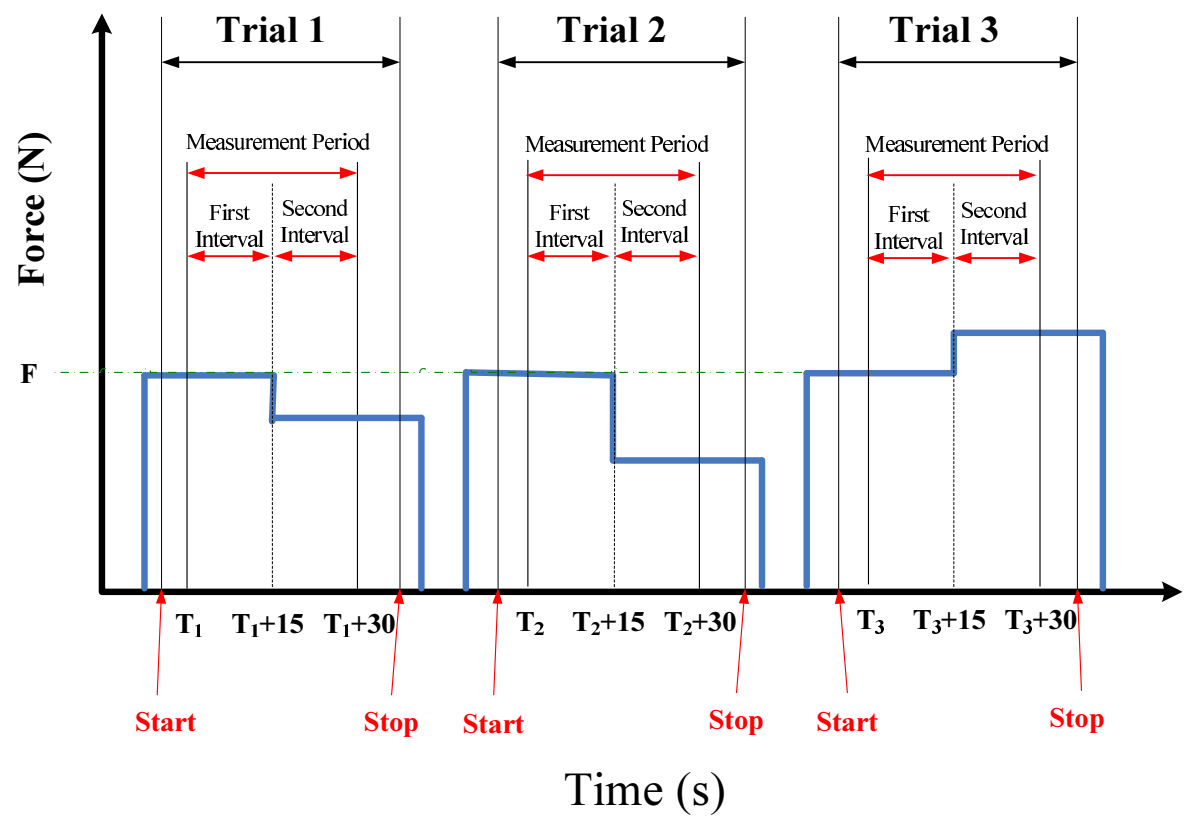

Fig. 12. The first three trials of an experiment(Times indicated are in seconds.)

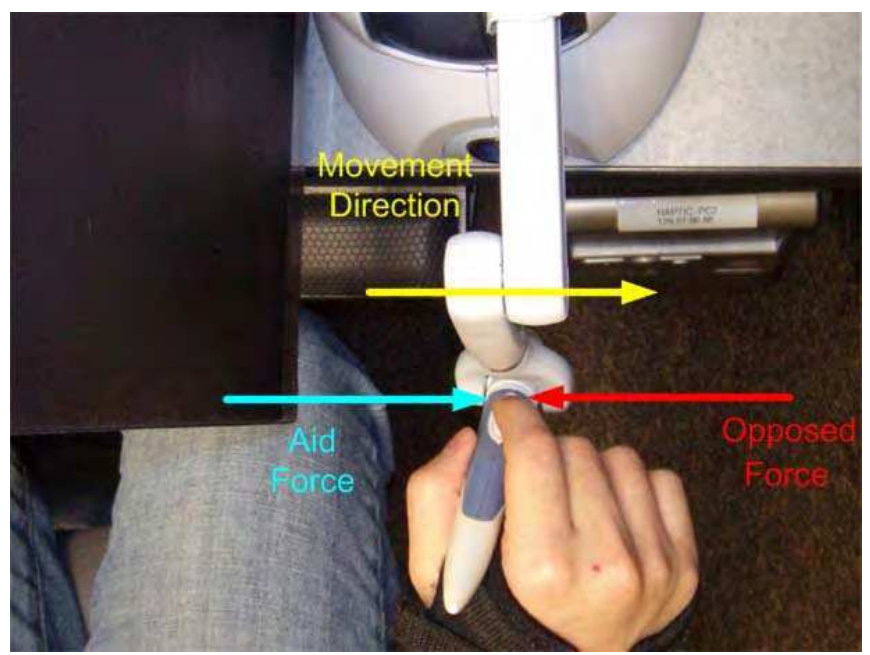

Fig. 13. The opposed and aid force vectors. 


\begin{tabular}{|c||c||c||c||c|}
\hline $\begin{array}{c}\text { Base force } \\
\text { Intensity }\end{array}$ & $\begin{array}{c}\text { Force } \\
\text { Inc./Dec. }\end{array}$ & $\begin{array}{c}\text { Mean } \\
\text { JND (\%) }\end{array}$ & $\begin{array}{c}\text { Standard } \\
\text { Deviation }\end{array}$ & $\begin{array}{c}\text { Standard } \\
\text { Error }\end{array}$ \\
\hline Low & Increment & 64 & 22.36 & 5.59 \\
\hline Low & Decrement & 43 & 17.47 & 4.37 \\
\hline Medium & Increment & 15 & 6.93 & 1.73 \\
\hline Medium & Decrement & 12 & 3.79 & 0.95 \\
\hline High & Increment & 10 & 3.72 & 0.93 \\
\hline High & Decrement & 11 & 3.76 & 0.94 \\
\hline
\end{tabular}

Table 2. The average force thresholds (\%), standard deviations, and standard errors for all levels of the base force and force increment/decrement.

The points at which the subject's response changes from yes to no or vice versa are called transition points. The direction of the force increasing/decreasing is reversed from increasing to decreasing, or vice versa at these points.

At the beginning of the experiment, the subject's responses might not be valid due to unfamiliarity with the type of force sensation. Thus, the first two transition points are neglected Cornsweet T. N. (1962). The force JND is the average values of the third transition point to the last one. To find the JND in \%, this force value should be divided by the base force.

\subsubsection{Task}

The subject engages in a task similar to the Fitts' task (Fitts P.M., 1954). During each trial, subjects are asked to tap the two targets (green rectangles) by moving their hand to left and right. Each time the ball is within one of the targets, a hit is scored by subjects. An overshoot error occurs if they pass the target. An undershoot error happens if they did not reach the target. Subjects are asked to score as many hits as they can and carry out the task as rapidly as possible and as accurately as possible for a predetermined duration.

Unlike the Fitts' task, subjects are required to maintain their hand velocity within a specified range. The reference velocity range is set by the experimenter to $0.16-0.20 \mathrm{~m} / \mathrm{s}$ based on the ability of a subject to carry out the task with an acceptable range of missing error (less than $15 \%$ ). The missing error equals the sum of overshoots and undershoots divided by the total hits, overshoots, and undershoots. A training session is delivered to help subjects to get familiar with the task. It is required for subjects that carry out the task with less than $15 \%$ missing error at the end of training session and prior to the beginning of the experiment.

In this setup, the colour of the ball is also determined based on the hand's velocity and the reference velocity to help a subject to keep the hand's velocity within the range. If the subject's velocity is within the range, the ball's colour is red; otherwise its colour is yellow. The mean velocity value is monitored as it does not rapidly change when the subject stops at the target. As shown in Figure 12, each trial has a measurement period, including two 15-second intervals (before and after force increment/decrement). The first interval is started when the experimenter ensures that the hand's velocity is within the reference range. The number of hits, overshoots, and undershoots are separately measured during each interval.

\subsection{Results and Discussion}

The average of JND values for all levels of the two factors are shown in Table 2 and Figure 14. 


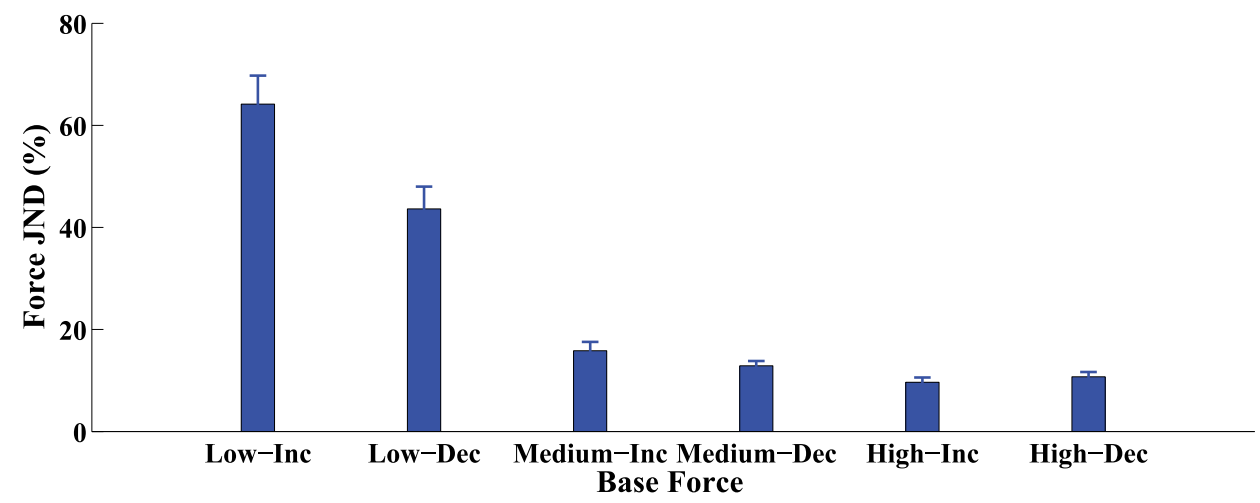

Fig. 14. The average and standard errors of force JND values for all subjects. (Inc $=$ Force Increment, Dec $=$ Force Decrement)

The result is analyzed using the repeated-measures (within subject) Analysis of Variance (ANOVA). The analysis is done at a significance level of 0.05 .

As shown in Figure 14, there are several trends present in the data. One trend shows that the base force intensity has a major effect on the force JND. The force JND significantly decreases by increasing the base force intensity. The figure also shows a significant difference between the force increment and decrement of the low base force. The difference decreases for the medium and high base forces, indicating that there is an interaction between the base force intensity and force increment/decrement. The results of a two way ANOVA, F $(2,30)=$ 12.28 and $\mathrm{p}<0.0001$, also confirm the interaction between the base force intensity and force increment/decrement.

The results of a post-hoc Tukey test also confirm the large difference between the force increment and decrement of the low base force $(\mathrm{p}<0.0001)$. This difference shows that the subjects notice the decrements of the low base force, Low-Dec, more easily than the increments, Low-Inc. This might be due to the fact that force is applied in the same direction of hand motion and the total resistive force is decreased on the subject's hand. This result rejects the null hypothesis in favour of $\mathrm{H}_{3}$ hypothesis. In other words, the upper and lower limens of low base force JND are not symmetric.

The results in Figure 14 show a Weber trend, which is explained in Section 3.1. The force JND is noticeably large for the low base forces and decreases for the medium and high base forces. The results of the post-hoc test show a significant difference between the JNDs of the low base inc/dec and the medium or high base inc/dec $(\mathrm{p}<0.0001)$. These results support the significant effect of the base force intensity on the force JND, rejecting the null hypothesis in favour of $\mathrm{H}_{2}$ hypothesis.

In the previous section, Section 4 , the force thresholds are determined with respect to a friction base force $(0.27 \mathrm{~N})$; however, that was for a different velocity range. To find the JNDs for the same velocity as the velocities implemented in this section, the JNDs for the friction are estimated based on a linear interpolation of JNDs for two ranges of velocities (0.12-0.15 and $0.22-0.28 \mathrm{~m} / \mathrm{s}$ ). The resulting force JNDs are $31.6 \%$ and $29.6 \%$ for the upper and lower limens of the friction base force. Figure 15 shows a Weber trend for the JNDs measured in the current and previous sections, confirming that the JNDs of small base forces are larger than high base 


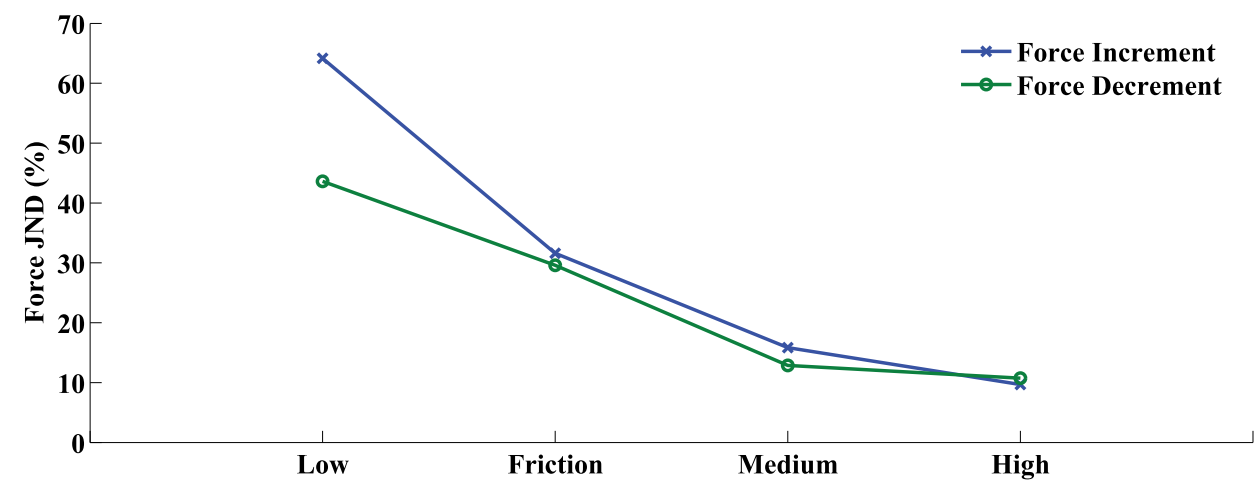

Fig. 15. The Weber's fraction for four base force intensities.

forces' JNDs. The JNDs for friction base forces are smaller than the low base force JNDs and greater than the medium base force JNDs.

Figure 15 does not show any significant difference between the force increment and decrement of the medium and high base forces. In other words, the upper and lower limens of JND are somewhat symmetric for medium and high base force intensities. The results of the post-hoc test also show no significant difference ( $\mathrm{p}=0.9433$ for medium and $\mathrm{p}=0.9995$ for high).

The results show that, for applications that require motion within a constant velocity range, the JNDs are in the extremely small base force region of the Weber's fraction. For example, the low base force JNDs (62\% and 38\%) are comparable with the JNDs measured by Raj et al. (1985), who found that the human sensitivity is very low for small weights (20-60 g). Their results (JNDs ranging between $89 \%$ and $35 \%$ ) indicate that as the base weights increases, JND decreases and remains relatively constant at weights above $200 \mathrm{~g}$.

The standard errors (or standard deviations) of the low base force JNDs are greater than the JNDs of the medium and high base force. This indicates that the subjects are more confident in their reports about the medium and high base force JNDs.

The medium and high base force JNDs (around 13\%) are very similar to the JNDs measured by Raj et al. (1985) who studied the ability of human subjects to discriminate between different magnitudes of weights. Their results show a JND of $12 \%-13 \%$ for relatively large base weights (80-200 g) lifted by the middle finger. The medium and high base force JNDs are also similar to the JNDs obtained by Jandura and Srinivasan (1994), who found $12.7 \%$ torque JND when the reference torque was $0.06 \mathrm{Nm}$.

The high base force JNDs (around 10\%) are very similar to the JNDs measured by other researchers (Pang et al., (1991); Jones L. A. (1989); and Allin et al., (2002)). They found JNDs in a range of $7 \%-10 \%$ for different muscle groups in hand and arm under various conditions. (Jones L. A., 1989), in a force matching experiment about the elbow, found a JND ranging between 5\% and 9\% over a range of different base forces. Pang et al. (1991) found a 5\% to $10 \%$ JND for pinching motions between the finger and thumb with a constant resisting force over base forces between 2.5 and $10 \mathrm{~N}$. The high base force JNDs are almost the same as the JND measured in a VE by Allin et al. (2002) using the PHANToM ${ }^{T M}$ Omni device. They found a $10 \%$ force JND on the index finger with a constant base force at $2.25 \mathrm{~N}$.

Our medium and high base force JNDs are much smaller than the JNDs obtained in a VE by Brewer et al. (2005) who found a $19.7 \%$ force JND (base force: $1.5 \mathrm{~N}$ ) for the index finger of 
young subjects (ages 18-35) and a 31\% force JND (base force: $2 \mathrm{~N}$ ) for elderly subjects (ages 61-80). They confirmed that their JNDs are relatively high and discussed reasons why their JND is larger than the JND in the literature such as the difference in the environment and tested joint, less subjects' training, and unfixed background dimensions.

\section{Conclusions and Future Directions}

This study reports the results of experimental research designed to investigate the limitations of human force perception when the user's hand moves in a haptic-enabled virtual environment (HEVE). The thresholds of force perception are measured with respect to the factors such as the user's hand velocity, the base force, and force increment/decrement.

The results of this study have provided a basis for which the integration of the force JNDs in the presence of velocity can be used to transmit compressed haptic data unbeknown to the user. The threshold of human force perception plays a significant role in the development of these techniques. This study investigates the impact of important factors on the force threshold that affect these techniques when the user's hand is in motion. These factors include the base force intensity, force increment/decrement, and velocity of the user's hand.

The results show that force JNDs depend on the user's hand velocity, the base force and the force increment/decrement. Thus, these variables must be incorporated in an efficient haptic data compression algorithm when the user's hand is in motion. For example, if a user's hand is in a faster motion, fewer haptic details are required to be stored, calculated or transmitted. The results indicate that haptic display developers cannot efficiently store or send the haptic data over the network when they are not aware of the previous applied force on the user's hand and if the forces increases or decreases. For example, the results and analysis of data in Section 5.3 show a Weber trend for the measured force JND, indicating that the force JND is significantly large for extremely small base forces and it decreases for the higher base forces. The results indicate that the friction of haptic devices should be taken into account in the design of compression methods. In addition, the variability of friction is important. The variability of the OMNI device's friction did not largely affect the process of measuring the force threshold because it was very small. However, the variability of friction would be large and more complicated for many haptic devices and should be considered in developing a compression technique.

The human factors issues that are raised by the results of the experiments may guide future studies. For instance, based on the results, the effect of the base force on the JND of the human force perception is dependent on the force increment/decrement. This indicates that the interaction of these two factors should be taken into consideration in the design of haptic display of VEs. In addition, although the upper and lower limens of JND are almost symmetric for the medium and high base forces, they are not symmetric for the low base force. In other words, the user is not equally sensitive to the increment or decrement of applied forces for all base forces.

Time is critical in the development of compression techniques. Thus, time-efficient methods are essentials to measure the required force thresholds. Many psychophysical studies (Gescheider G.A. (1997)) have required long-term experiments to study human perception. For example in Pang et al., (1991) each experiment took hours with an average of 2048 trials for one experimental condition. In addition, the adaptation to force is also problematic in very long experimental sessions. In our study, each level of the experiment is completed within roughly a 50-minute session with 48 trials. The IS method takes less time compared to other methods because only a few stimuli values that are far above or below threshold are 
presented. As a result, a suitable compromise is found between the robust results and time to obtain specific data relevant to the development of perception-based compression methods.

\section{References}

Allin S., Matsuoka Y., and Klatzky R. L. (2002). Measuring Just Noticeable Differences for Haptic Force Feedback: Implications for Rehabilitation, Symposium on Haptic Interfaces for Virtual Environment and Teleoperator Systems pp. 299-302.

Berkelman, P. and Ji M. (2006). Effects of Friction Parameters on Completion Times for Sustained Planar Positioning Tasks with a Haptic Interface, Proceedings of the 2006 IEEE/RSJ International Conference on Intelligent Robots and Systems pp. 1115-1120. Beijing, China.

Bernstein R. S., and Gravel J. S. (1990). Method for determining hearing sensitivity in infants; The interweaving staircase procedure, Journal of the American Academy of Audiology 1: 138-145.

Bhaskaran V., Konstantinides K. (1999). Image and Video Compression Standards Algorithms and Architectures, second edn, Kluwer Academic Publishers, USA.

Brandenburg K. (1999). MP3 and ACC explained, Proceedings of the AES 17th International Conference on High Quality Audio Coding . Florence, Italy.

Brewer B.R., Fagan M., Klatzky R.L. and Matsuoka Y. (2005). Perceptual limits for a robotic rehabilitation environment using visual feedback distortion, Neural Systems and Rehabilitation Engineering, IEEE Transactions on [see also IEEE Trans. on Rehabilitation Engineering] 13(1): 1-11.

Brisben A. J., Hsiao S. S., and Johnson K. O. (1999). Detection of vibration transmitted through an object grasped in the hand, Journal of Neurophysiology 81: 1548-1558.

Colgate J.E. and Brown J.M. (1994). Factors Affecting the Z-width of a Haptic Display, Proceeding of the IEEE Int'l Conf. Robotics and Automation pp. 3205-3210.

Cornsweet T. N. (1962). The Staircase-Method in Psychophysics, The American Journal of Psychology 75(3): 485-491.

Diolaiti, N., Niemeyer, G., Barbagli, F., Salisbury, J.K., and Melchiorri, C. (2005). The effect of quantization and Coulomb friction on the stability of haptic rendering, Proceedings of the First Joint Eurohaptics Conference and Symposium on Haptic Interfaces for Virtual Environment and Teleoperator Systems pp. 237-246. 18-20 March.

Fitts P.M. (1954). The information capacity of the human motor system in controlling the amplitude of movement, Journal of Experimental Psychology 47: 381-391.

Gersho A. (1994). Advances in speech and audio compression, 900-918 .

Gescheider G.A. (1997). Psychophysics: The Fundamentals, 3rd edn, Lawrence Erlbaum Associates.

Hinterseer P. and Steinbach E. (2005). Psychophysically Motivated Compression of Haptic Data, In Proceedings of the Joint International COE/HAM - SFB453 Workshop on Human Adaptive Mechatronics and High Fidelity Telepresence .

Hinterseer P. and Steinbach E. (2006). A Psychophysically Motivated Compression Approach for 3D Haptic Data, In 14th Symposium on Haptic Interfaces for Virtual Environment and Teleoperator Systems pp. p.35-41.

Hinterseer P., Steinbach E. and Chaudhuri S. (2006). Model-based Data Compression for 3D Virtual Haptic Teleinteraction, IEEE International Conference on Consumer Electronics, ICCE 2006. 
Hinterseer P., Steinbach E., Chaudhuri S. (2006). Perception-Based Compression of haptic Data Streams Using Kalman Filters, IEEE Intern. Conf. On Acoustics, Speech and Signal Processing pp. pV-473-V-476.

Hinterseer P., Steinbach E., Hirche S., and Buss M. (2005). A novel, psychophysically motivated transmission approach for haptic data streams in telepresence and teleaction systems, In Proceedings of the IEEE International Conference on Acoustics, Speech, and Signal Pro-cessing pp. 1097-1100. Philadelphia, PA, USA.

Jandura L. and Srinivasan M. A. (1994). Experiments on Human Performance in Torque Discrimination and Control, Proc. of the ASME Dynamic Systems and Control Division 551: 369-375.

Jones L. A. (1989). Matching forces: Constant errors and differential thresholds, Perception 18(5): 681-687.

Karnopp D. (1985). Computer simulation of stick-slip friction in mechanical dynamic systems, ASME Journal of dynamic Systems, Measurement and Control 107: 100-103.

Kuehl R. O. (2000). Design of experiments : statistical principles of research design and analysis, 2nd edn, Pacific Grove, CA ; London : Duxbury/Thomson Learning.

Kuschel M., Kremer P., Hirche S., and Buss M. (2006). Lossy data reduction methods for haptic telepresence systems, Proceedings 2006 IEEE International Conference on Robotics and Automation ICRA 2006 . Las Vegas.

Lederman S.J., Klatzky R.L., Hamilton C.L. and Ramsay G.I. (1999). Perceiving roughness via a rigid probe: Psychophysical effects of exploration speed and mode of touch, Haptics-e (Electronic Journal of Haptics Research) 1(1): 1-20.

McLaughlin M.L., Joao P. Hespanha, and Gaurav S.S. (2002). Touch in virtual environments: haptics and the design of interactive systems, Prentice Hall.

Miano J. (1999). Compressed Image File Formats: JPEG, PNG, GIF, XBM, BMP, New York: ACM Press.

Pang X., Tan H.Z. and Durlach N. (1991). Manual discrimination of force using active finger motion, Perception \& Psychophysics 49(6): 531-540.

Raj D.V., Ingty K., and Devanandan M.S. (1985). Weight appreciation in the hand in normal subjects and in patients with leprous neuropathy, Brain 108(1): 95-102.

Reis, H. \& Judd, C. (2000). Handbook of Research Methods in Social and Personality Psychology, Cambridge University Press. ISBN 0521559030, 9780521559034.

Schiffman, H.R. (2000). Sensation and perception : an integrated approach, 5th edn, New York : Wiley.

Sensable Technologies Inc. (n.d.). http:/ / www.sensable.com.

Shahabi C., Ortega A., and Kolahdouzan M.R. (2002). A Comparison of Different Haptic Compression Techniques, Multimedia and Expo .

Srinivasan M. and Basgodan C. (1997). Haptics in Virtual Environments: Taxonomy, Research Status, and Challenges, Computers and Graphics 21(4): 393-404.

Zadeh M.H., Wang D. and Kubica E. (2008). Perception-Based Lossy Haptic Compression Considerations for Velocity-Based Interactions, Multimedia Systems Journal 13(4): 275282. 


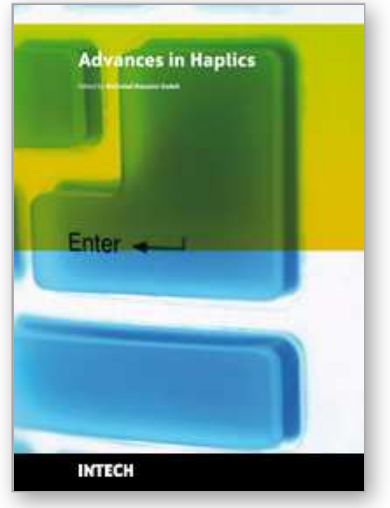

\author{
Advances in Haptics \\ Edited by Mehrdad Hosseini Zadeh
}

ISBN 978-953-307-093-3

Hard cover, 722 pages

Publisher InTech

Published online 01, April, 2010

Published in print edition April, 2010

Haptic interfaces are divided into two main categories: force feedback and tactile. Force feedback interfaces are used to explore and modify remote/virtual objects in three physical dimensions in applications including computer-aided design, computer-assisted surgery, and computer-aided assembly. Tactile interfaces deal with surface properties such as roughness, smoothness, and temperature. Haptic research is intrinsically multidisciplinary, incorporating computer science/engineering, control, robotics, psychophysics, and human motor control. By extending the scope of research in haptics, advances can be achieved in existing applications such as computer-aided design (CAD), tele-surgery, rehabilitation, scientific visualization, robot-assisted surgery, authentication, and graphical user interfaces (GUI), to name a few. Advances in Haptics presents a number of recent contributions to the field of haptics. Authors from around the world present the results of their research on various issues in the field of haptics.

\title{
How to reference
}

In order to correctly reference this scholarly work, feel free to copy and paste the following:

Mehrdad Hosseini Zadeh, David Wang and Eric Kubica (2010). Factors Affecting the Perception-Based Compression of Haptic Data, Advances in Haptics, Mehrdad Hosseini Zadeh (Ed.), ISBN: 978-953-307-093-3, InTech, Available from: http://www.intechopen.com/books/advances-in-haptics/factors-affecting-theperception-based-compression-of-haptic-data

\section{INTECH}

open science | open minds

\section{InTech Europe}

University Campus STeP Ri

Slavka Krautzeka 83/A

51000 Rijeka, Croatia

Phone: +385 (51) 770447

Fax: +385 (51) 686166

www.intechopen.com

\section{InTech China}

Unit 405, Office Block, Hotel Equatorial Shanghai No.65, Yan An Road (West), Shanghai, 200040, China 中国上海市延安西路65号上海国际贵都大饭店办公楼405单元 Phone: +86-21-62489820

Fax: $+86-21-62489821$ 
(C) 2010 The Author(s). Licensee IntechOpen. This chapter is distributed under the terms of the Creative Commons Attribution-NonCommercialShareAlike-3.0 License, which permits use, distribution and reproduction for non-commercial purposes, provided the original is properly cited and derivative works building on this content are distributed under the same license. 